\title{
Performance à 25 ans de quelques provenances de mélèze laricin (Larix laricina (Du Roi) Koch) sur sols hydromorphes
}

\author{
LE Pâques, C Périnot \\ INRA, station d'amélioration des arbres forestiers, F-45160 Ardon, France
}

(Reçu le 24 février 1993; accepté le 17 décembre 1993)

\begin{abstract}
Résumé - L'étude à 25 ans d'un test tri-stationnel de provenances de mélèze laricin montre la bonne adaptation de cette espèce (survie comprise entre $60 \%$ et $91 \%$ ) sur des sols hydromorphes considérés comme marginaux pour l'épicéa commun. Sa productivité moyenne estimée à 50 ans oscillerait entre 4 et $10 \mathrm{~m} 3 / \mathrm{ha} /$ an suivant le site. Bien que le milieu de test soit la principale source de variabilité, des différences statistiquement significatives ont été mises en évidence entre provenances pour les principaux caractères étudiés. Hormis pour les caractères de qualité du bois, les performances des provenances apparaissent stables entre sites. Compte tenu des fortes liaisons juvéniles/adultes, il semble dès à présent qu'une sélection des meilleurs individus pour la vigueur à 25 ans soit fiable dès l'âge de 7 ans (depuis la graine). D'autre part, alors qu'au niveau provenance vigueur et infradensité ne sont pas liées défavorablement, il apparaît difficile de trouver des provenances à forte croissance et bonne forme du fût. Cependant, il est à noter que, quel que soit le site, le mélèze laricin souffre de défauts de forme sévères qui limitent son intérêt. Le choix des provenances pour le reboisement est brièvement discuté ainsi que l'incorporation de cette essence dans le programme d'amélioration génétique des mélèzes.
\end{abstract}

mélèze laricin / Larix laricina / provenance / croissance / qualité du bois

Summary - Results at $\mathbf{2 5}$ years of a multisite provenance trial of tamarack in France. Eight provenances of tamarack from the southeastern part of its natural range have been compared on 3 waterlogged sites. Results at 25 years (from seed) are presented. The good adaptation of tamarak to wet soils is confirmed in this study as the survival varies from 60 to $91 \%$ and the yield class estimated at 50 years ranges from 4 to $10 \mathrm{~m}^{3} / \mathrm{ha}$ /year according to sites. Apart from site, which is the main source of variation, the provenances also differ significantly for most characters and are mainly stable over sites, except for wood quality traits. Due to high juvenile-mature correlations, selection for total height and stem volume at 25 years (from seed) is efficient as early as 7 years using total height. At the provenance level, vigour and wood density are not unfavourably correlated; in contrast, it is difficult to find provenances both vigorous and with a good stem-form quality. Nevertheless, whatever the site, tamarack suffers from severe stem-form defects, such as crookedness, which limit its interest compared to other larches. Choice of provenances for reforestation of waterlogged sites is discussed briefly as well as the place of tamarack in the larch breeding programme.

tamarack / Larix laricina / provenance / growth / wood quality 


\section{INTRODUCTION}

L'aire naturelle du mélèze laricin (Larix laricina (Du Roi) Koch) - tamarack en anglais - est une des plus étendues parmi celles des conifères américains (fig 1). Elle occupe le nord des États-Unis et le Canada entre les latitudes $40^{\circ}$ et $64^{\circ} \mathrm{N}$, à la limite de la végétation forestière, et les longitudes $52^{\circ}$ et $160^{\circ} \mathrm{W}$. Sur ce vaste territoire, l'espèce rencontre des conditions écologiques très variées, en particulier de climat et de sol. Plusieurs études de variabilité par comparaison de populations en test de provenances (Jeffers, 1975 ; Cech et al, 1977) ou par marqueurs enzymatiques (Cheliak et al, 1988) montrent l'existence d'une différenciation génétique très grande de l'espèce avec une diversité génétique accrue dans le bassin des Grands Lacs.

Une des caractéristiques essentielles de l'espèce est sa grande tolérance vis-à-vis des conditions d'humidité et d'aération du sol puisqu'elle se rencontre depuis les sols minéraux sableux secs jusqu'aux sols organiques de tourbière à engorgement permanent (Fowells, 1965).

Sa tolérance vis-à-vis des sols hydromorphes a retenu l'attention des améliorateurs qui cherchent à valoriser ce caractère exclusif du mélèze laricin, soit en sélection récurrente simple comme espèce pure, soit en hybridation interspécifique avec les mélèzes d'Europe et du Japon (Ferrand, 1986 ; Pâques, 1992).

Hormis quelques exemplaires introduits pour certains dès 1770 (en Grande-Bretagne) dans les parcs et arboreta, l'espèce est mal connue en Europe et en France. Des essais de comparaison de provenances ont cependant été installés dès 1955 en Allemagne du Nord (Winkler, 1973) et 1967 en Suède (Martinsson, 1992).

L'objectif de cet article est de faire la synthèse d'un test multistationnel de comparaison de provenances installé en France à partir de 1968, en examinant en particulier l'intérêt de cette essence pour les reboisements sur sols hydromorphes.

\section{MATÉRIEL ET MÉTHODES}

\section{Provenances testées}

L'immense aire de répartition du mélèze laricin et la diversité des conditions écologiques rencontrées suggèrent une très grande variabilité génétique. Pour installer ce premier test de comparaison de provenances en France - test dont l'ambition n'était pas d'explorer l'ensemble de la variabilité infraspécifique mais plutôt d'étudier le comportement de cette essence sur sol hydromorphe -- un choix a priori de populations a été fait dans une zone aux conditions écologiques supposées voisines des conditions françaises.

L'objectif étant de favoriser le caractère d'adaptation avant tout autre critère de vigueur ou de forme, les populations du nord et de l'ouest de l'aire aux climats boréal ou continental ont été supposées mal adaptées et ont donc été abandonnées. Huit provenances ont pu être obtenues. Elles occupent le sud-est de l'aire naturelle, à des latitudes comparables à celles de la France (fig 1). Leur localisation précise est donnée au tableau I.

Le matériel a été semé en avril 1966 et repiqué au printemps 1967 à la pépinière de l'INRANancy (Meurthe-et-Moselle) avant d'être installé en forêt aux printemps 1968 et 1969.

\section{Sites et dispositifs expérimentaux}

Trois sites expérimentaux ont été retenus, 2 en forêt domaniale de Larfeuil sur le plateau de Millevaches (Limousin) et 1 en forêt communale d'Épinal (plateau lorrain). Une description écologique est donnée au tableau II ainsi que les modalités de plantation et d'entretien des dispositifs. De plus amples détails sont fournis par Périnot (1992).

Les 3 sites correspondent à des sols acides de tourbière à hydromorphie marquée, soit sur plateau : Larfeuil non drainé $\left(L_{n d}\right)$, soit en fond de vallée : Épinal $(E)$ et Larfeuil drainé $\left(L_{d}\right)$. Ce der- 


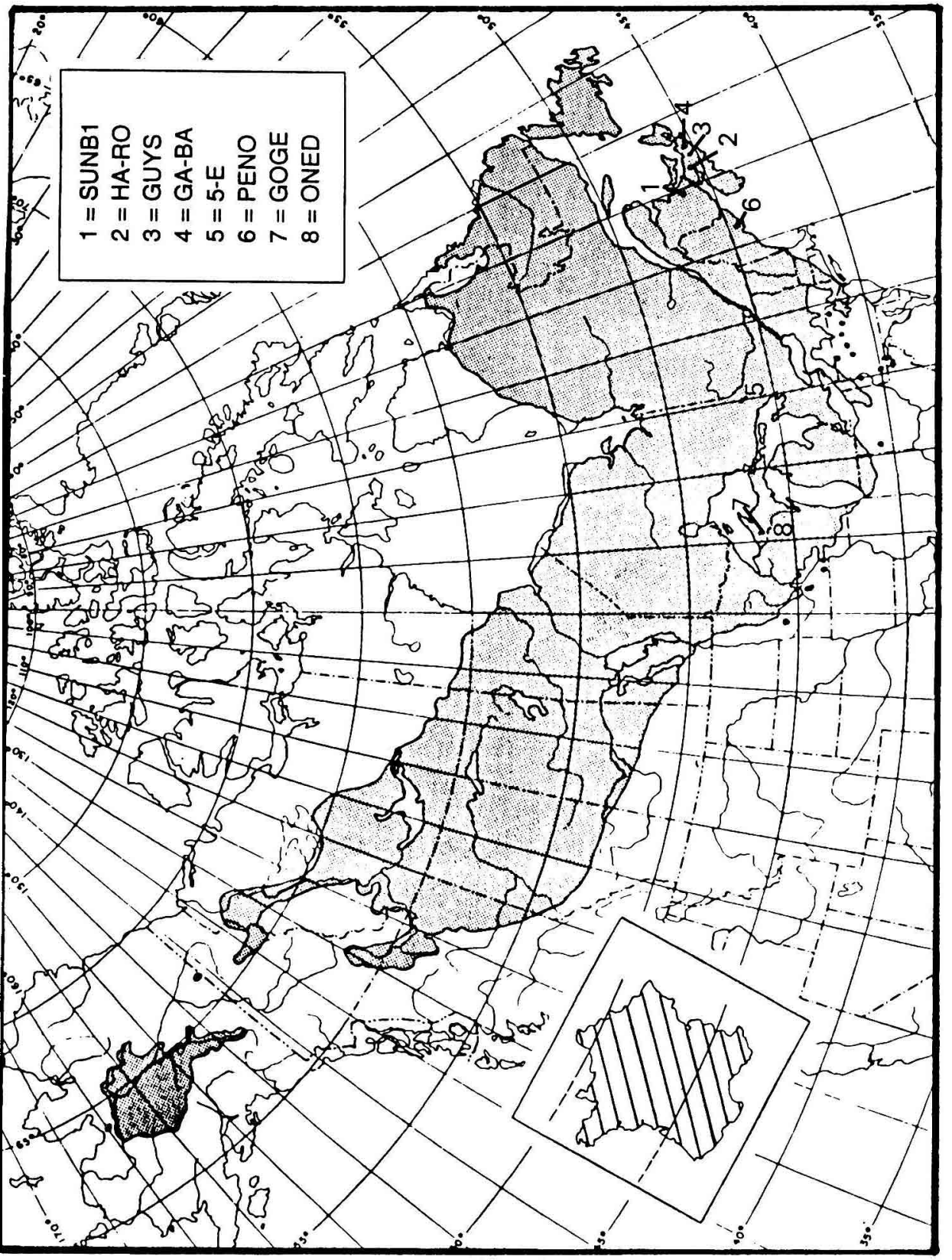

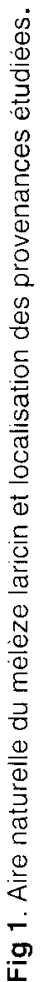


Tableau I. Description des populations étudiées.

\begin{tabular}{|c|c|c|c|c|c|}
\hline Code & Provenance & Région administrative & Latitude & Longitude & Altitude $(m$ \\
\hline--- & $-T_{-}$ & …..... & & - & \\
\hline UNB1 & Sunbury 1 & Nouveau-Brunswick (CND) & $46^{\circ} \mathrm{N}$ & $66^{\circ} 22 ' W$ & 60 \\
\hline $2 \mathrm{HA}-\mathrm{RO}$ & Ahoushagon Road & Nouveau-Brunswick (CND) & $45^{\circ} 59^{\prime} \mathrm{N}$ & $64^{\circ} 21^{\prime} \mathrm{W}$ & 15 \\
\hline 3 GUYS & Guysborough & Nouvelle-Écosse (CND) & $45^{\circ} 40^{\prime} \mathrm{N}$ & $62^{\circ} 40^{\prime} \mathrm{W}$ & $<90$ \\
\hline 4 GA-BA & Garden of Eden Barren & Nouvelle-Écosse (CND) & $45^{\circ} 20^{\prime} \mathrm{N}$ & $62^{\circ} 15^{\prime} \mathrm{W}$ & 150 \\
\hline $55 \mathrm{E}$ & Zone $5 \mathrm{E}$ & Ontario (CND) & $46^{\circ} \mathrm{N}$ & $80^{\circ} \mathrm{W}$ & - \\
\hline 6 PENO & Penobscot & Maine (USA) & $44^{\circ} 51^{\prime} \mathrm{N}$ & $68^{\circ} 37^{\prime} W$ & 36 \\
\hline 7 GOGE & Gogebic & Michigan (USA) & $46^{\circ} 15^{\prime} \mathrm{N}$ & $89^{\circ} 10^{\prime} \mathrm{W}$ & 480 \\
\hline 8 ONED & Oneida & Wisconsin (USA) & $45^{\circ} 46^{\prime} \mathrm{N}$ & $89^{\circ} 12^{\prime} \mathrm{W}$ & 360 \\
\hline
\end{tabular}

Tableau II. Caractéristiques des sites d'expérimentation

Larfeuil draine

(Ld)

$\begin{array}{ll}\text { Localisation } & (\mathrm{Ld}) \\ \text { Département } & \text { Corrèze } \\ \text { Latitude } & 45^{\circ} 32^{\prime} \mathrm{N} \\ \text { Longitude } & 1^{\circ} 58^{\prime} \mathrm{E} \\ \text { Altitude } & 815 \mathrm{~m}\end{array}$

Climat

Pluv annuelle

Sol

Type

$\mathrm{pH}$

Topographie

Exposition

Relief

Préparation sol

Plants

Type

Date semis

Dispositif

Modèle

Nbre blocs

Parc unitaire

$S$ (ares)

Nbre plants

Plantation

Date

Densité

Entretiens

$1400 \mathrm{~mm}$

tourbière

4,25-5,25

sud-ouest

fond vallon

$1+1$

$04 / 1966$ 6

9 plants

18,9

378

$10 / 04 / 1968$

$2 \times 2,5 \mathrm{~m}$

aucun
1965/66 drainage

potets à la pioche

décapage $05 \times 06 \mathrm{~m}$

$1+1$

$04 / 1966$

bloc aléatoire incomplet bloc aléatoire incomplet

$1400 \mathrm{~mm}$

tourbière

$4,15-5,5$

plat

potets à la pioche

décapage $0.5 \times 0.6 \mathrm{~m}$

9 plants

15,1

378

$9 / 04 / 1968$

$2 \times 2 \mathrm{~m}$

aucun
Larfeuil non drainé

Épinal

(Lnd)

Corrèze

$45^{\circ} 32^{\prime} \mathrm{N}$

25-42 plants
$1+2$

$04 / 1966$

1968/69 coupe rase potets tarrière

(E)

Vosges

$48^{\circ} 05^{\prime} \mathrm{N}$

$6^{\circ} 34^{\prime} \mathrm{E}$

$350 \mathrm{~m}$

$1100 \mathrm{~mm}$

tourbière

sud/ sud-est

cuvette

bloc aléatoire incomplet

5

33,8

864

$27 / 05 / 1969$

$2 \times 2 \mathrm{~m}$

1978 dégagement

1983 éclaircie sélective

1990 éclaircie systématique 
nier site a été drainé 2 ans avant plantation. Le site de Larfeuil non drainé se caractérise par un tapis continu de molinie. Par suite des risques de gelées tardives et du niveau d'hydromorphie élevé, les 3 sites représentent des stations extrêmes pour le reboisement en épicéa commun.

Un schéma expérimental en blocs aléatoires incomplets a été utilisé sur les 3 sites avec un nombre de blocs et de plants par parcelle unitaire variables suivant les sites (tableau II). La distance de plantation est de $2 \times 2 \mathrm{~m}$ sauf à Larfeuil drainé où les canaux de drainage ont imposé une plantation à $2,5 \times 2 \mathrm{~m}$.

La plantation a eu lieu en avril 1968 sur les 2 sites de Larfeuil et en mai 1969 à Épinal à partir du même matériel. Depuis, seule la plantation d'Épinal, la plus vigoureuse, a été éclaircie à 2 reprises, de façon sélective en 1983 et systématique en 1990

\section{Mesures et observations}

Les caractères étudiés concernent d'une part l'adaptation, estimée par le pourcentage de survie à 25 ans depuis la graine (codé $S$ ), et d'autre part la croissance et des paramètres extrinsèques et intrinsèques de qualité du bois.

La hauteur totale de la tige (codée $\mathrm{H}$ ) a été mesurée en pépinière à 2 ans puis en forêt à 3,4 , $5,6,7,12,13$ et 25 ans ainsi que la circonférence à $1,30 \mathrm{~m}(\mathrm{C})$ à 25 ans ; la pousse cumulée hors crise de plantation $(\mathrm{Pc})$ a été calculée à partir de $\mathrm{H} 25-\mathrm{H} 7$. Le volume total de la tige a été utilisé comme mesure de la biomasse totale et a été estimé approximativement par la formule du cône à partir de $\mathrm{C}$ et $\mathrm{H}$.

La forme de la tige a été caractérisée par 4 paramètres : une notation subjective de rectitude-flexuosité suivant une échelle de 1 (très flexueux) à 5 (droit) (codée Rc), d'où est déduite une fréquence de tiges flexueuses (FI) correspondant à $R c<4$; la présence/absence de fourches et la fréquence de tiges avec ce défaut (Fo) et une observation de la présence/absence de double pied (Dp). Le défaut de courbure basale très fréquent sur les autres espèces de mélèze n'a pas été rencontré sur laricin et a donc été abandonné.

Trois paramètres de qualité du bois ont été évalués : une mesure de pénétration au pilodyn
(6 joules, aiguille de $2 \mathrm{~mm}$ ) à $1,30 \mathrm{~m}$ sur tous les arbres, sur écorce et face sud-est : Pi ; l'infradensité déterminée par la méthode de Keylwerth (1954) sur 2 demi-carottes par arbre diamétralement opposées et prélevées à $1,30 \mathrm{~m}$ sur un échantillon de 20 arbres/provenance (ID) ; une mesure sur carotte de la longueur du rayon et du duramen pour en estimer sa proportion (DU). Ces paramètres de qualité du bois ont été étudiés sur les sites de Larfeuil drainé et d'Épinal.

Une description méthodologique plus détaillée est fournie par Périnot (1992) pour l'ensemble des caractères étudiés.

\section{ANALYSES STATISTIQUES}

Une analyse de variance multi-caractères a été conduite grâce au logiciel Anvarm de la chaîne Amance (Bachacou et al, 1981), séparément sur les 3 sites pour tester l'existence éventuelle de différences significatives entre provenances. Le modèle [1] a été utilisé pour $\mathrm{H}, \mathrm{Pc}, \mathrm{C}, \mathrm{Rc}$, et $\mathrm{Pi}$; le modèle simplifié [2] a été retenu pour ID et DU compte tenu de l'échantillonnage limité et déséquilibré par bloc :

$$
\begin{gathered}
Y_{i j k}=\mu+P_{i}+B_{j}+P B_{i j}+\varepsilon_{i j k} \\
Y_{i j}=\mu+P_{i}+\varepsilon_{i j}
\end{gathered}
$$

avec $P_{i}=$ effet de la provenance $; ; B_{j}=$ effet du bloc $\mathrm{j} ; P B_{i j}=$ interaction provenance $\mathrm{i} \mathrm{x}$ bloc $\mathbf{j} ; \varepsilon_{i j}(k)=$ résidu.

Les facteurs sont considérés comme aléatoires. Compte tenu du nombre variable de répétitions, les performances moyennes des provenances ajustées aux effets «bloc» ont été ensuite comparées par la méthode de Bonferroni (Bachacou et al, 1981).

Pour les caractères qualitatifs $(\mathrm{S}, \mathrm{FI}, \mathrm{FO}$ et $D p$ ), le test 2 l ou $G$ de Sokal et Rohlf (1981) a été utilisé pour tester l'homogénéité de réponse des provenances.

La stabilité des provenances inter-sites a été estimée après ajustement aux effets "site" par le test non paramétrique $S_{2}$ de 
Huhn (Nassar et Huhn, 1987), basé sur la variance des rangs des provenances intersites.

\section{RÉSULTATS}

\section{Performances générales}

Les performances moyennes sur chacun des 3 sites sont résumées au tableau III.

Le taux de survie à 25 ans le plus élevé est enregistré à Larfeuil drainé $(91,0 \%)$. II reste très satisfaisant sur le site non drainé $(82,8 \%)$ mais baisse jusqu'à $62,9 \%$ à Épinal, suggérant une moins bonne adaptation sur ce dernier site. En fait, un examen détaillé du site dÉpinal suggère l'existence dans le dispositif d'une zone en amorce de pente plus superficielle et plus sèche où la mortalité est élevée. Ailleurs, dans la zone fraîche, la survie est équivalente à celle observée sur les autres sites.

Pour la vigueur, des différences de conditions de croissance très marquées existent entre les 3 sites. À Épinal, la hauteur totale qui atteint $13,5 \mathrm{~m}$ à 25 ans est pratiquement

Tableau III. Performances moyennes à 25 ans (coefficients de variation entre parenthèses) sur les 3 sites et tests $\mathrm{F}$ ou $2 \mathrm{l}$ sur le facteur provenances.

\begin{tabular}{|c|c|c|c|c|c|c|}
\hline \multirow{3}{*}{$\begin{array}{l}\text { Effectif mesuré } \\
\text { Variable }\end{array}$} & \multicolumn{4}{|c|}{ Larfeuil } & \multicolumn{2}{|c|}{ Épinal (E) } \\
\hline & \multicolumn{2}{|c|}{$\begin{array}{l}\text { Drainé }(L d) \\
\quad 295\end{array}$} & \multicolumn{2}{|c|}{$\begin{array}{c}\text { Non drainé }(L n d) \\
265\end{array}$} & \multicolumn{2}{|l|}{185} \\
\hline & Moyenne & $F / 2 I$ & Moyenne & $F / 2 I$ & Moyenne & $F / 2 I$ \\
\hline Survie (\%) & 91,0 & * & 82,8 & * & 62,9 & * \\
\hline Hauteur totale $(\mathrm{m})$ & $\begin{array}{l}9,91 \\
(23,0)\end{array}$ & $* * *$ & $\begin{array}{l}6,82 \\
(38,5)\end{array}$ & ns & $\begin{array}{l}13,5 \\
(16,2)\end{array}$ & $\star \star \star \star ~$ \\
\hline Pousse cumulée (m) & $\begin{array}{l}8,23 \\
(22,6)\end{array}$ & 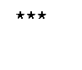 & $\begin{array}{l}5,95 \\
(41,0)\end{array}$ & ns & $\begin{array}{l}11,13 \\
(18,5)\end{array}$ & $\star * *$ \\
\hline Circonférence $(\mathrm{cm})$ & $\begin{array}{l}36,5 \\
(34,0)\end{array}$ & $\star * *$ & $\begin{array}{l}23,8 \\
(47,4)\end{array}$ & ns & $\begin{array}{l}45,9 \\
(23,2)\end{array}$ & ** \\
\hline Volume $\left(\mathrm{dm}^{3}\right)$ & $\begin{array}{l}44,0 \\
(71,6)\end{array}$ & $* \star *$ & $\begin{array}{l}16,2 \\
(114,0)\end{array}$ & ns & $\begin{array}{l}83,0 \\
(54,5)\end{array}$ & ** \\
\hline Rectitude (note) & $\begin{array}{l}3,6 \\
(30,7)\end{array}$ & $\star * *$ & $\begin{array}{l}3,3 \\
(30,8)\end{array}$ & $\star \star \star$ & $\begin{array}{l}3,5 \\
(29,6)\end{array}$ & 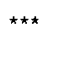 \\
\hline Tiges flexueuses (\%) & 43,4 & * & 54,7 & * & 43,2 & ns \\
\hline Fourche $(\%)$ & 28,5 & * & 20,8 & ns & 9,7 & * \\
\hline Double-pied (\%) & 3,7 & ns & 17,7 & * & 0,0 & ns \\
\hline Pilodyn (mm) & $\begin{array}{l}18,7 \\
(27,4)\end{array}$ & 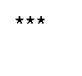 & - & - & $\begin{array}{l}21,5 \\
(9,6)\end{array}$ & $\star \star$ \\
\hline Infradensité $\left(\mathrm{kg} / \mathrm{m}^{3}\right)$ & $\begin{array}{l}462,8 \\
(6,0)\end{array}$ & $\star \star \star$ & - & - & $\begin{array}{l}445,0 \\
(6,8)\end{array}$ & ns \\
\hline Duramen (\%) & $\begin{array}{l}58,8 \\
(16,1)\end{array}$ & * & - & - & $\begin{array}{l}57,5 \\
(20,6)\end{array}$ & ns \\
\hline
\end{tabular}


le double de celle de Larfeuil non drainé $(6,8 \mathrm{~m})$, site à forte concurrence de molinie ; à Larfeuil drainé, les performances sont intermédiaires $(9,9 \mathrm{~m})$. Comme lillustre la figure 2, la phase d'installation qui correspond à la crise de plantation est assez longue sur les 3 sites puisqu'elle dure jusqu'à 7 ans, soit jusqu'à 5 ans après plantation. Après cette phase, la croissance est très active et la différence de croissance entre sites s'accentue très nettement avec le temps.

Pour la circonférence, le classement des sites reste le même : Épinal, Larfeuil drainé et Larfeuil non drainé avec respectivement $45,9,36,5$ et $23,8 \mathrm{~cm}$. En conséquence, comparé à Épinal, le site le plus favorable, le volume individuel moyen à 25 ans sur le site de Larfeuil drainé représente $53,0 \%$ de celui à Épinal et celui à Larfeuil non drainé $19,5 \%$ seulement.

À titre de comparaison, une placette de Pinus uncinata a été plantée conjointement à côté des 2 dispositifs de Larfeuil. Cette

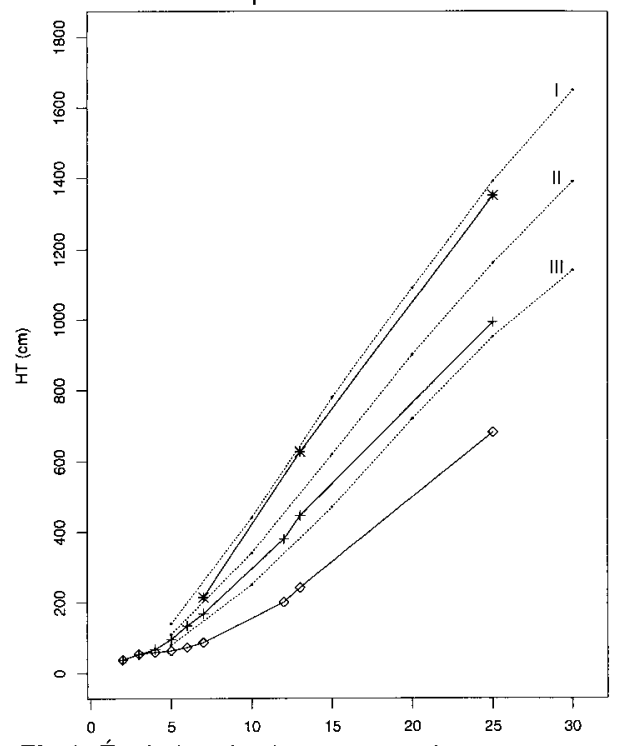

Fig 2. Évolution des hauteurs totales moyennes (H) sur les 3 sites et courbes des classes de productivité I, II, III du mélèze d'Europe selon Schober (1946). * Épinal ; + Larfeuil drainé ; $\diamond$ Larfeuil non drainé. espèce que l'on rencontre naturellement sur tourbière a été utilisée comme essence témoin. Sur la parcelle drainée, les performances à 25 ans s'élèvent à $5,2( \pm 0,8) \mathrm{m}$ pour la hauteur totale et à $31,6( \pm 7,1) \mathrm{cm}$ pour la circonférence et représentent $52 \%$ et $86 \%$ respectivement des performances du mélèze laricin. Sur la parcelle non drainée, la hauteur totale atteint $5,1( \pm 1,0) \mathrm{m}$, soit $75 \%$ de celle du mélèze et la circonférence : $36,0( \pm 9,6) \mathrm{cm}$, soit $151 \%$ de celle du mélèze.

Suivant les tables anglaises de production (Hamilton et Christie, 1971) pour le mélèze d'Europe et en admettant que cellesci soient transposables au mélèze laricin, le site d'Épinal se situerait à un niveau de productivité compris entre 9 et $10 \mathrm{~m}^{3 / \mathrm{ha}} / \mathrm{an}$ (à 50 ans), celui de Larfeuil drainé à 6 $\mathrm{m}^{3} / \mathrm{ha}$ /an et celui de Larfeuil non drainé tomberait en-dessous de $4 \mathrm{~m}^{3} / \mathrm{ha}$ /an. Suivant les tables allemandes de Schober (1946, in Erted, 1962), cela correspondrait respectivement aux classes I, II/III et <III de productivité pour le mélèze d'Europe.

Par ailleurs, comme l'indiquent les valeurs élevées des coefficients de variation (tableau III), la variabilité individuelle est aussi la plus forte sur le site de Larfeuil non drainé. Ce dernier apparaît donc comme le site le plus médiocre en terme de performances moyennes mais aussi d'hétérogénéité de performances.

Winkler (1973) observe également en Allemagne du Nord une très forte variabilité de comportement de site à site avec des hauteurs totales à 13 ans comprises entre 1 et $6 \mathrm{~m}$. Dans les meilleures stations, la production du laricin est largement supérieure à celle du mélèze d'Europe ( $>$ classe I de Schober).

Des défauts de forme sévères sont notés sur les tiges dans les 3 sites. Les performances sont très proches entre sites pour la rectitude de la tige ( $R c$ et $F 1$ ) mais contrastées pour la fourchaison ( $F o$ ) et le défaut de double-pied (Dp). Sur les 3 sites y com- 
pris Épinal où une éclaircie sélective a eu lieu en 1983, presque 1 tige sur 2 présente un défaut de flexuosité marqué (note inférieure ou égale à 3 ). D'autre part, la proportion d'arbres fourchus est élevée à Larfeuil (drainé et non drainé) (>20\%), de même que la proportion de tiges avec double-pied sur le site de Larfeuil non drainé $(17,7 \%)$. Globalement, les tiges ne présentent pas de courbure basale, défaut caractéristique chez les mélèzes d'Europe et du Japon.

La mauvaise forme du mélèze laricin a été notée également par Winkler (1973) qui estime entre 60 et $90 \%$ la fréquence de tiges défectueuses. II insiste en revanche sur la finesse de la branchaison, qualité observée sur les 3 sites étudiés.

Pour les 3 paramètres de qualité du bois, les sites de Larfeuil drainé et d'Épinal sont très voisins. Le site de Larfeuil à croissance plus lente donnerait cependant un bois légèrement plus dense que celui d'Épinal comme l'indiquent les valeurs de pénétration au pilodyn : 18,7 et $21,5 \mathrm{~mm}$ et d'infradensité : 462,8 et $445,0 \mathrm{~kg} / \mathrm{m}^{3}$. Ce dernier caractère (de même que $\mathrm{Pi}$ à Épinal) présente une faible variabilité individuelle : $\mathrm{CV}<7 \%$. À titre de comparaison, à 15 ans, dans un test de provenances installé en Bretagne, l'infradensité pour le mélèze d'Europe, du Japon et leur hybride était sensiblement plus faible avec des valeurs respectivement de 413,362 et $402 \mathrm{~kg} / \mathrm{m}^{3}$ (Bastien et Keller, 1980).

La proportion de duramen est équivalente sur les 2 sites; elle représente à 25 ans respectivement $58,8 \%$ et $57,5 \%$ de la longueur du rayon.

\section{Performances moyennes des provenances}

Sur chacun des 3 sites, des différences significatives entre provenances sont observées pour les caractères d'adaptation, de vigueur, de forme et de qualité du bois (tableau III). Quelques exceptions intéressantes sont cependant à noter. D'une part, l'absence de différence significative entre provenances pour les caractères de vigueur à Larfeuil non drainé suggère le peu d'intérêt de ce site aux conditions de croissance limite pour discriminer des populations. D'autre part, certains autres caractères présentent également peu de variabilité au niveau provenance : fréquences de tiges flexueuses et de double-pied, infradensité et proportion de duramen à Épinal, fourchaison à Larfeuil non drainé et fréquence de double-pied à Larfeuil drainé.

Les performances moyennes des provenances à 25 ans sont donnés site par site aux tableaux IV et $\mathrm{V}$.

Pour la survie, les meilleures provenances sont respectivement Guysborough, Garden of Eden Barren et Penobscot à Larfeuil drainé, non drainé et Épinal ; pour la vigueur, Garden of Eden Barren ou Guysborough suivant les caractères sur les 3 sites (fig 3a); pour la rectitude du fût, Zone $5 \mathrm{E}$ à Larfeuil et Gogebic à Épinal ; pour la faible fréquence de tiges flexueuses, Zone $5 \mathrm{E}$ à Larfeuil non drainé et Oneida sur les autres sites (fig $3 b$ ). Pour les paramètres de qualité du bois respectivement à Larfeuil drainé et à Epinal, Zone $5 \mathrm{E}$ et Gogebic pour la faible pénétration au pilodyn ; Gogebic et Guysborough pour leur forte infradensité et Ahoushagon Road et Sunbury pour le pourcentage élevé de duramen.

Comparée aux valeurs sur les sites de Larfeuil drainé et d'Épinal, la valeur élevée des coefficients de variation intra-provenances pour les caractères de vigueur est particulièrement à noter sur le site non drainé de Larfeuil qui semble induire une forte hétérogénéité de performances et masque ainsi les différences entre provenances.

D'autre part, malgré l'existence de différences significatives entre provenances pour plusieurs caractères, les tests de compa- 
Tableau IV. Performances moyennes à 25 ans (ajustées aux effets bloc) des provenances, CV intra-provenance (en \%, entre parenthèses) et test $S_{2}$ de Huhn sur les sites de Larfeuil drainé $\left(L_{d}\right)$ et non drainé $\left(L_{n d}\right)$ et d'Épinal $(E)$.

\begin{tabular}{|c|c|c|c|c|c|c|c|c|c|c|c|}
\hline \multirow[t]{2}{*}{ Provenances } & \multicolumn{3}{|c|}{ Survie (\%) } & \multicolumn{2}{|c|}{ Hauteur (m) } & \multicolumn{3}{|c|}{ Rectitude (note) } & \multicolumn{3}{|c|}{ Tiges flexueuses(\%) } \\
\hline & $L_{d}$ & $L_{n d}$ & $E$ & $L_{n d}$ & $E$ & $L_{d}$ & $L_{n d}$ & $E$ & $L_{d}$ & $L_{n d}$ & $E$ \\
\hline Sunbury I & $\begin{array}{l}96,3 \\
-\end{array}$ & $\begin{array}{l}88,9 \\
-\end{array}$ & $\begin{array}{l}69,3 \\
-\end{array}$ & $\begin{array}{ll}10,2 & 6,9 \\
(12,4) & (22,1)\end{array}$ & $\begin{array}{l}12,6 \\
(10,8)\end{array}$ & $\begin{array}{l}3,2 \\
(25,9)\end{array}$ & $\begin{array}{l}3,1 \\
(26,3)\end{array}$ & $\begin{array}{l}3,1 \\
(30,9)\end{array}$ & 66,0 & $\underline{60,5}$ & $\stackrel{45,5}{-}$ \\
\hline Ahoushagon RD & $\begin{array}{l}92,6 \\
-\end{array}$ & $\begin{array}{l}88,9 \\
-\end{array}$ & $\begin{array}{l}66,7 \\
-\end{array}$ & $\begin{array}{ll}9,9 & 6,9 \\
(11,3) & (20,9)\end{array}$ & $\begin{array}{l}13,1 \\
(9,1)\end{array}$ & $\begin{array}{l}2,8 \\
(29,8)\end{array}$ & $\begin{array}{l}2,7 \\
(21,6\end{array}$ & $\begin{array}{l}3,0 \\
(24,0)\end{array}$ & $\begin{array}{l}52,2 \\
-\end{array}$ & $\begin{array}{l}86,4 \\
-\end{array}$ & $\begin{array}{l}47,0 \\
-\end{array}$ \\
\hline Guysborough & $\begin{array}{l}98,1 \\
-\end{array}$ & $\begin{array}{l}92,6 \\
-\end{array}$ & $\begin{array}{l}56,4 \\
-\end{array}$ & $\begin{array}{l}9,4 \quad 7,0 \\
(14,4)(24,1)\end{array}$ & $\begin{array}{l}14,3 \\
(8,9)\end{array}$ & $\begin{array}{l}2,6 \\
(29,2)\end{array}$ & $\begin{array}{l}2,9 \\
(30,5\end{array}$ & $\begin{array}{l}3,3 \\
(29,4)\end{array}$ & $\begin{array}{l}84,0 \\
-\end{array}$ & $\begin{array}{l}73,5 \\
-\end{array}$ & $\begin{array}{l}49,5 \\
-\end{array}$ \\
\hline Garden Eden & $\begin{array}{l}92,6 \\
-\end{array}$ & $\begin{array}{l}100,0 \\
-\end{array}$ & $\begin{array}{l}51,3 \\
-\end{array}$ & $\begin{array}{l}11,4 \quad 7,3 \\
(14,3)(26,0)\end{array}$ & $\begin{array}{l}14,1 \\
(8,1)\end{array}$ & $\begin{array}{l}2,8 \\
(29,8)\end{array}$ & $\begin{array}{l}3,0 \\
(26,4)\end{array}$ & $\begin{array}{l}3,6 \\
(17,5)\end{array}$ & $\begin{array}{l}70,8 \\
-\end{array}$ & $\begin{array}{l}72,0 \\
-\end{array}$ & $\begin{array}{l}51,2 \\
-\end{array}$ \\
\hline Zone $5 \mathrm{E}$ & $\underline{72,2}$ & $\begin{array}{l}72,2 \\
-\end{array}$ & $\begin{array}{l}69,6 \\
-\end{array}$ & $\begin{array}{ll}8,8 & 6,1 \\
(17,5) & (27,7)\end{array}$ & $\begin{array}{l}13,3 \\
(10,7)\end{array}$ & $\begin{array}{l}4,5 \\
(13,8)\end{array}$ & $\begin{array}{l}4,5 \\
(27,5)\end{array}$ & $\begin{array}{l}3,9 \\
(19,5)\end{array}$ & $\begin{array}{l}18,2 \\
-\end{array}$ & $\begin{array}{l}31,2 \\
-\end{array}$ & $\begin{array}{l}44,6 \\
-\end{array}$ \\
\hline Penobscot & $\begin{array}{l}94,4 \\
-\end{array}$ & $\begin{array}{l}77,8 \\
-\end{array}$ & $\begin{array}{l}71,8 \\
-\end{array}$ & $\begin{array}{ll}10,9 & 6,7 \\
(12,1) & (19,9)\end{array}$ & $\begin{array}{l}13,7 \\
(8,2)\end{array}$ & $\begin{array}{l}4,0 \\
(24,1)\end{array}$ & $\begin{array}{l}3,8 \\
(18,5)\end{array}$ & $\begin{array}{l}3,6 \\
(20,4)\end{array}$ & $\begin{array}{l}32,0 \\
-\end{array}$ & $\begin{array}{l}32,5 \\
-\end{array}$ & $\begin{array}{l}48,7 \\
-\end{array}$ \\
\hline Gogebic & $\begin{array}{l}88,9 \\
-\end{array}$ & $\begin{array}{l}74,1 \\
-\end{array}$ & $\begin{array}{l}65,5 \\
-\end{array}$ & $\begin{array}{ll}8,5 & 6,5 \\
(18,4) & (22,9)\end{array}$ & $\begin{array}{l}13,9 \\
(7,6)\end{array}$ & $\begin{array}{l}4,3 \\
(16,8)\end{array}$ & $\begin{array}{l}4,0 \\
(26,7)\end{array}$ & $\begin{array}{l}4,3 \\
(30,3)\end{array}$ & 16,3 & $\frac{42,4}{-}$ & $\frac{31,5}{-}$ \\
\hline Oneida & $\begin{array}{l}94,4 \\
-\end{array}$ & $\begin{array}{l}79,6 \\
-\end{array}$ & $\begin{array}{l}51,3 \\
-\end{array}$ & $\begin{array}{l}10,2 \quad 7,2 \\
(14,2)(21,5)\end{array}$ & $\begin{array}{l}13,3 \\
(10,9)\end{array}$ & $\begin{array}{l}4,4 \\
(13,9)\end{array}$ & $\begin{array}{l}3,5 \\
(23,7)\end{array}$ & $\begin{array}{l}4,2 \\
(16,7)\end{array}$ & $\frac{8,2}{-}$ & $\begin{array}{l}50,0 \\
-\end{array}$ & $\begin{array}{l}30,7 \\
-\end{array}$ \\
\hline$S_{2}$ & & ns & & ns & & & ns & & & ns & \\
\hline
\end{tabular}

${ }^{\star,}{ }^{\star \star},{ }^{\star \star \star}:$ significatif pour $\alpha=0,05,0,01$ et 0,001

raison de moyennes de Bonferroni permettent peu de discriminer les provenances entre elles (fig 3a, b et c). Malgré le caractère très conservateur de ce test, cette relativement faible différenciation des provenances intra-site a été observée également par Winkler (1973) en Allemagne et Martinsson (1992) en Suède. II est particulièrement intéressant de la contraster avec la forte dispersion des performances intersites induites par les différences stationnelles.

\section{Stabilité inter-sites}

Comme le montrent les tableaux IV et $\mathrm{V}$ pour les caractères principaux, le test de stabilité de Huhn ne permet pas de mettre en évidence une instabilité des classements des provenances sur les 3 sites pour les caractères d'adaptation (S), de vigueur $(H$, $\mathrm{Pc}, \mathrm{C}$ ) et de forme (Rc, F1). En revanche, d'assez fortes différences de classement des provenances entre sites ont été mises 
Tableau V. Performances moyennes à 25 ans (ajustées aux effets bloc) des provenances, $\mathrm{CV}$ intra-provenance (en \%, entre parenthèses) et test $\mathrm{S}_{2}$ de Huhn (suite).

\begin{tabular}{|c|c|c|c|c|c|c|}
\hline \multirow[b]{2}{*}{ Provenances } & \multicolumn{2}{|c|}{$\begin{array}{l}\text { Pilodyn } \\
(\mathrm{mm})\end{array}$} & \multicolumn{2}{|c|}{$\begin{array}{c}\text { Infradensité } \\
\left(\mathrm{kg} / \mathrm{m}^{3}\right)\end{array}$} & \multicolumn{2}{|c|}{$\begin{array}{c}\text { Duramen } \\
(\%)\end{array}$} \\
\hline & $L d$ & $E$ & $L d$ & E & $L d$ & $E$ \\
\hline & & & & & & \\
\hline Sunbury I & $\begin{array}{l}20,5 \\
(13,0)\end{array}$ & $\begin{array}{l}20,9 \\
(9,7)\end{array}$ & $\begin{array}{l}473,8 \\
(4,6)\end{array}$ & $\begin{array}{l}432,4 \\
(10,2)\end{array}$ & $\begin{array}{l}59,4 \\
-\end{array}$ & $\begin{array}{l}63,0 \\
-\end{array}$ \\
\hline Ahoushagon RD & $\begin{array}{l}20,1 \\
(8,9)\end{array}$ & $\begin{array}{l}21,2 \\
(6,0)\end{array}$ & $\begin{array}{l}466,3 \\
(4,8)\end{array}$ & $\begin{array}{l}447,3 \\
(8,7)\end{array}$ & $\begin{array}{l}65,3 \\
-\end{array}$ & $\frac{61,8}{-}$ \\
\hline Guysborough & $\begin{array}{l}20,1 \\
(12,6)\end{array}$ & $\begin{array}{l}22,1 \\
(10,1)\end{array}$ & $\begin{array}{l}476,9 \\
(6,8)\end{array}$ & $\begin{array}{l}446,6 \\
(6,5)\end{array}$ & $\begin{array}{l}59,0 \\
-\end{array}$ & $\begin{array}{l}58,6 \\
-\end{array}$ \\
\hline Garden Eden & $\begin{array}{l}21,3 \\
(10,2)\end{array}$ & $\begin{array}{l}21,2 \\
(9,1)\end{array}$ & $\begin{array}{l}471,8 \\
(5,4)\end{array}$ & $\begin{array}{l}447,2 \\
(5,6)\end{array}$ & $\begin{array}{l}56,9 \\
-\end{array}$ & $\begin{array}{l}58,5 \\
-\end{array}$ \\
\hline Zone $5 \mathrm{E}$ & $\begin{array}{l}18,4 \\
(12,3)\end{array}$ & $\begin{array}{l}21,2 \\
(11,0)\end{array}$ & $\begin{array}{l}459,9 \\
(6,1)\end{array}$ & $\begin{array}{l}453,9 \\
(5,2)\end{array}$ & $\begin{array}{l}53,0 \\
-\end{array}$ & $\begin{array}{l}55,6 \\
-\end{array}$ \\
\hline Penobscot & $\begin{array}{l}20,8 \\
(10,7)\end{array}$ & $\begin{array}{l}21,7 \\
(9,8)\end{array}$ & $\begin{array}{l}465,3 \\
(3,6)\end{array}$ & $\begin{array}{l}439,3 \\
(5,1)\end{array}$ & $\begin{array}{l}58,5 \\
-\end{array}$ & $\begin{array}{l}52,1 \\
-\end{array}$ \\
\hline Gogebic & $\begin{array}{l}18,4 \\
(9,6)\end{array}$ & $\begin{array}{l}20,1 \\
(6,4)\end{array}$ & $\begin{array}{l}451,6 \\
(7,3)\end{array}$ & $\begin{array}{l}458,5 \\
(4,9)\end{array}$ & $\begin{array}{l}58,0 \\
-\end{array}$ & $\begin{array}{l}55,3 \\
-\end{array}$ \\
\hline Oneida & $\begin{array}{l}20,5 \\
(10,2)\end{array}$ & $\begin{array}{l}20,9 \\
(9,2)\end{array}$ & $\begin{array}{l}442,1 \\
(5,8)\end{array}$ & $\begin{array}{l}442,8 \\
(7,7)\end{array}$ & $\begin{array}{l}60,1 \\
-\end{array}$ & $\begin{array}{l}54,5 \\
-\end{array}$ \\
\hline $\mathrm{S}_{2}$ & & & * & & & \\
\hline
\end{tabular}

en évidence pour la fréquence de fourchaison (Fo) et de double-pied (Dp) et les paramètres de qualité du bois (PI, ID, DU).

Les provenances les plus instables sont Garden of Eden Barren et Gogebic pour la fourchaison; Garden of Eden Barren et Oneida pour la fréquence de double-pied; Garden of Eden Barren et Zone 5 E pour la mesure de pilodyn ; Sunbury et Gogebic pour l'infradensité et Sunbury et Penobscot pour le pourcentage de duramen.

\section{Liaisons entre caractères}

Des liaisons positives, fortes et très hautement significatives existent entre les 3 carac- tères de vigueur: hauteur totale, circonférence (tableau VI) et longueur de la pousse cumulée.

Par ailleurs, une représentation graphique des liaisons âge-âge entre 2 et 25 ans est donnée à la figure 4 pour les hauteurs totales intermédiaires en fonction de la hauteur totale $(\mathrm{H})$ et du volume individuel moyen $(\mathrm{V})$ à 25 ans. Seuls les 2 sites de Larfeuil sont figurés car le site d'Épinal comportait trop peu de points. Cette représentation permet de repérer l'âge à partir duquel une bonne prédiction des performances à un âge adulte peut être espérée, âge à partir duquel une sélection juvénile sera efficace.

Pour la hauteur totale, la liaison très faible (Larfeuil drainé) ou négative (Larfeuil non 
Volume individuel moyen ( $\mathrm{dm} 3$ )

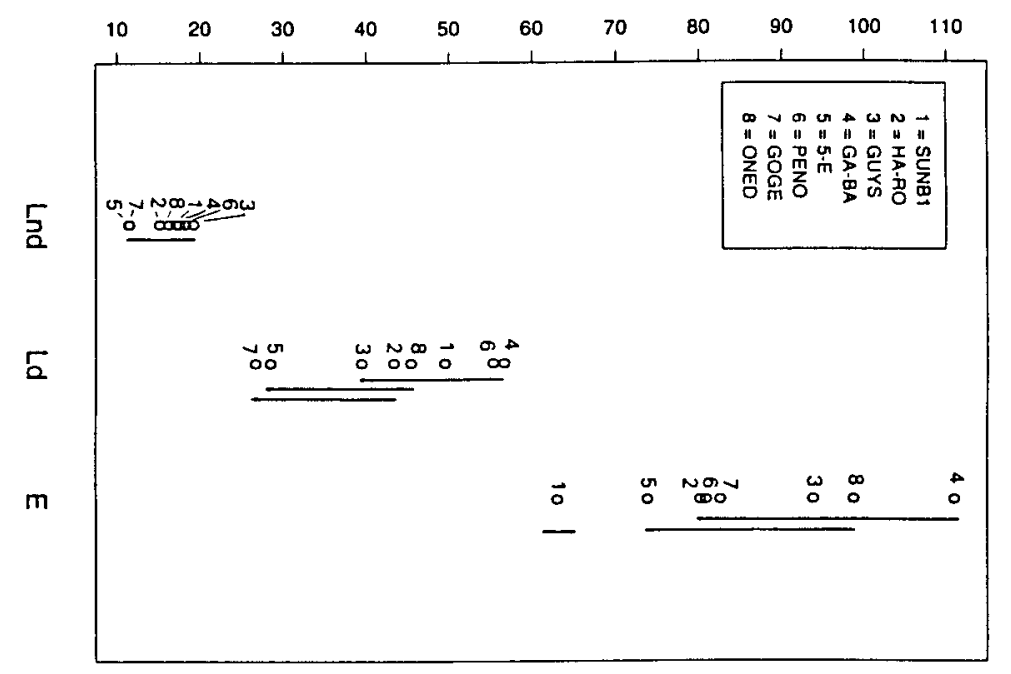

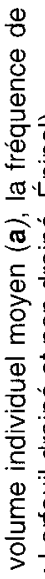

$\%$ tiges flexueuses

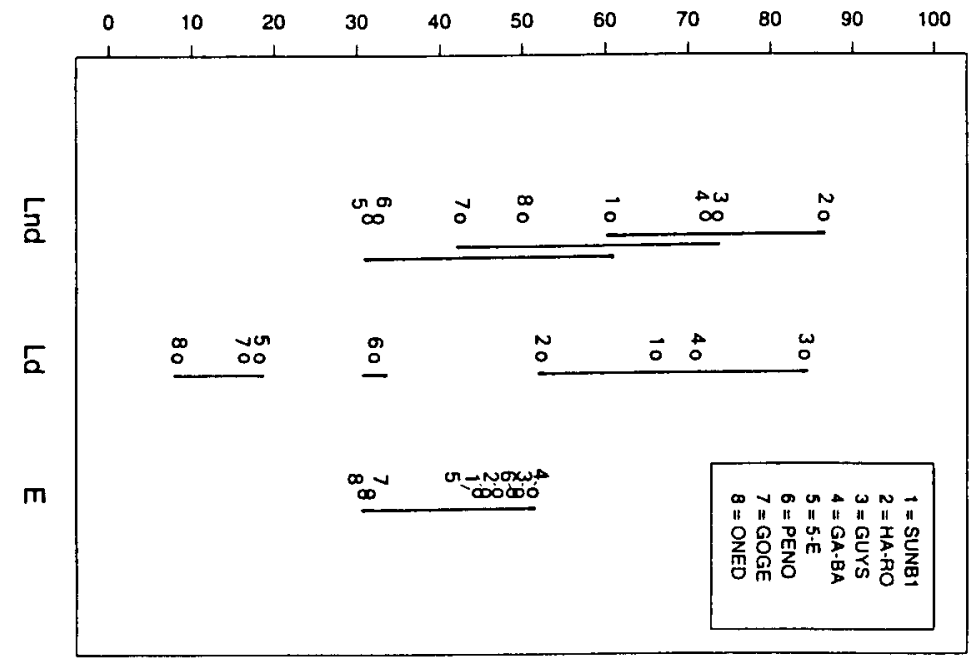

Infradensite $(\mathrm{kg} / \mathrm{m} 3)$

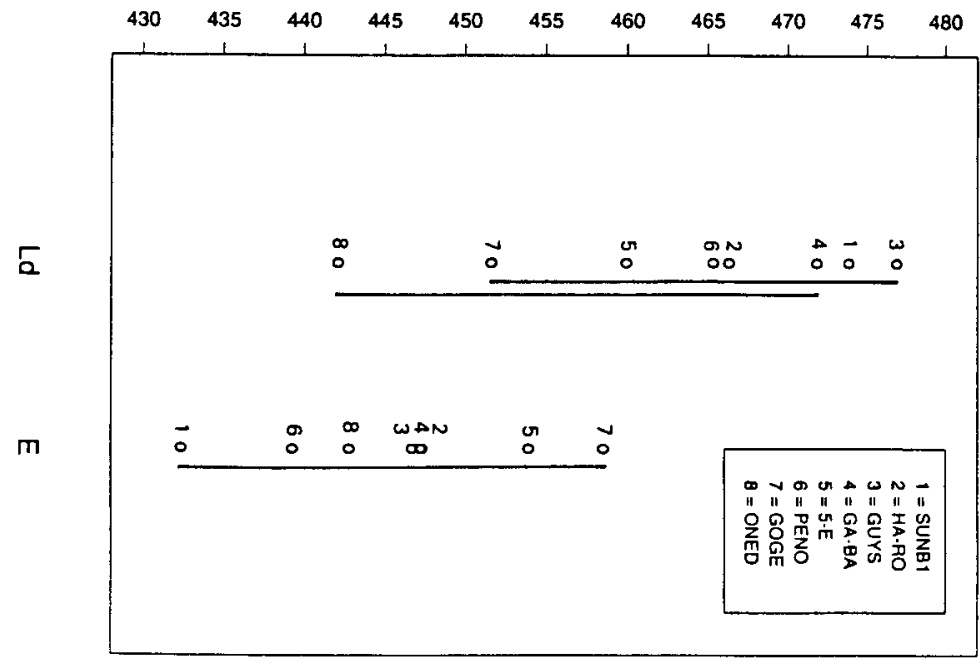

言

0

递

蛋 in

응

$\Phi \overline{\frac{N}{O}}$

क

$\stackrel{\infty}{\Phi}$

ब.



过

宅

हैํํㅇ

$\frac{1}{8} \frac{0}{8}$

용

造

is

$\stackrel{\Phi}{\frac{\Phi}{4}} \stackrel{0}{\circ}$

क्ष

$\stackrel{\Phi}{\underline{5}}$

$\overline{0}$

莣

으은

䨔

을

엉

ن

ㅇำ

if

लि

은음 
Tableau VI. Corrélations phénotypiques au niveau individuel (dessus diagonale) et corrélations de rang au niveau moyennes de provenances (dessous diagonale) entre caractères à 25 ans pour chacun des 3 sites $\left(L_{d}, L_{n d}\right.$ et $E$ : Larfeuil drainé et non drainé, Épinal) (valeurs $\times 1000$ ).

\begin{tabular}{|c|c|c|c|c|c|c|}
\hline Sites & $\begin{array}{l}\text { Hauteur } \\
(H)\end{array}$ & $\begin{array}{c}\text { Circonférence } \\
\text { (C) }\end{array}$ & $\begin{array}{l}\text { Rectitude } \\
\quad(R c)\end{array}$ & $\begin{array}{l}\text { Pilodyn } \\
\quad(P i)\end{array}$ & $\begin{array}{l}\text { Infradensité } \\
\text { (ID) }\end{array}$ & $\begin{array}{l}\text { Duramen } \\
(D U)\end{array}$ \\
\hline $\begin{array}{c}H L_{d} \\
L_{\text {nd }} \\
E\end{array}$ & & $\begin{array}{l}854^{\star \star \star} \\
881^{\star \star \star \star} \\
625^{\star \star \star}\end{array}$ & $\begin{array}{l}14^{\text {ns }} \\
156^{*} \\
-88^{\text {ns }}\end{array}$ & $\begin{array}{l}608^{\star * *} \\
- \\
200^{\star *}\end{array}$ & $\begin{array}{c}-210^{*} \\
- \\
41^{n s}\end{array}$ & $\begin{array}{l}377^{\star \star \star} \\
- \\
36^{n s}\end{array}$ \\
\hline $\begin{array}{c}C \mathrm{~L}_{\mathrm{d}} \\
\mathrm{L}_{\text {nd }} \\
\mathrm{E}\end{array}$ & $\begin{array}{l}976^{\star *} \\
850^{\star} \\
667^{\text {ns }}\end{array}$ & & $\begin{array}{l}\text { ons } \\
94^{\text {ns }} \\
37^{\text {ns }}\end{array}$ & $\begin{array}{l}733^{\star \star \star} \\
- \\
522^{\star \star \star}\end{array}$ & $\begin{array}{l}219^{\star *} \\
-\overline{294^{*}}\end{array}$ & $\begin{array}{l}453^{\star \star \star} \\
- \\
488^{\star \star \star}\end{array}$ \\
\hline $\begin{array}{c}R c L_{d} \\
L_{\text {nd }} \\
E\end{array}$ & $\begin{array}{r}-286^{\text {ns }} \\
-691^{\text {ns }} \\
286^{\text {ns }}\end{array}$ & $\begin{array}{l}-357^{n s} \\
-898^{*} \\
-95^{n s}\end{array}$ & & $\begin{array}{c}-40^{\mathrm{ns}} \\
- \\
157^{\mathrm{ns}}\end{array}$ & $\begin{array}{c}-197^{\star} \\
- \\
-265^{n s}\end{array}$ & $\begin{array}{c}-125^{\text {ns }} \\
-\end{array}$ \\
\hline $\begin{array}{c}P i L_{d} \\
L_{n d} \\
E\end{array}$ & $\begin{array}{l}976^{* *} \\
- \\
476^{n s}\end{array}$ & $\begin{array}{l}952^{\star} \\
- \\
738^{\star}\end{array}$ & $\begin{array}{c}-333^{n s} \\
- \\
-524^{n s}\end{array}$ & & $\begin{array}{c}-268^{\star \star \star} \\
- \\
-344^{\star}\end{array}$ & $\begin{array}{c}229^{\star *} \\
- \\
184^{\mathrm{ns}}\end{array}$ \\
\hline $\begin{array}{c}\text { ID } L_{d} \\
L_{n d} \\
E\end{array}$ & $\begin{array}{c}167^{\text {ns }} \\
- \\
310^{\text {ns }}\end{array}$ & $\begin{array}{c}310^{n s} \\
- \\
-119^{n s}\end{array}$ & $\begin{array}{c}-857^{\star} \\
- \\
357^{\mathrm{ns}}\end{array}$ & $\begin{array}{c}143^{\text {ns }} \\
- \\
-262^{\text {ns }}\end{array}$ & & $\begin{array}{c}-151^{\text {ns }} \\
- \\
-315^{\star}\end{array}$ \\
\hline $\begin{array}{c}D U L_{d} \\
L_{\text {nd }} \\
E\end{array}$ & $\begin{array}{c}143^{n s} \\
- \\
-286^{n s}\end{array}$ & $\begin{array}{c}119^{n s} \\
- \\
-238^{n s}\end{array}$ & $\begin{array}{c}-238^{\mathrm{ns}} \\
- \\
-810^{*}\end{array}$ & $\begin{array}{c}191^{\mathrm{ns}} \\
- \\
119^{\mathrm{ns}}\end{array}$ & $\begin{array}{c}95^{\mathrm{ns}} \\
- \\
-71^{\mathrm{ns}}\end{array}$ & \\
\hline
\end{tabular}

drainé) avant 5 ans se resserre peu à peu pour atteindre la valeur de 1 à 25 ans. Les 2 courbes présentent un point d'inflexion majeur entre 6 et 7 ans et une bonne prédiction de la hauteur à 25 ans peut ainsi être obtenue dès 12 ans $\left(r_{12-25}>0,78\right)$. Pour le volume individuel moyen à 25 ans, une très bonne liaison avec la hauteur totale est atteinte dès 7 ans à Larfeuil drainé $\left(r_{7-25}=\right.$ 0,73 ), soit 5 ans après plantation ; sur le site non drainé, cet âge est retardé puisqu'il faut attendre l'âge de 12 ans pour atteindre une liaison équivalente avec V. À Épinal également, $12-13$ ans seraient nécessaires pour prédire les performances à 25 ans.

De manière semblable, Riemenschneider et Jeffers (1980) observent, dans 2 tests de provenances dans le nord du Wisconsin, une liaison forte entre hauteurs totales à 7-8 ans et $13-14$ ans $(r=0,81-0,92)$, qui confirme nos résultats. Clairement, les performances en pépinière sont un mauvais prédicteur des performances ultérieures en forêt. La nature des 2 milieux est évidemment très différente.

Les liaisons au niveau individuel entre caractères de vigueur $(\mathrm{H}, \mathrm{C})$ et de forme (Rc) sont positives ou négatives (tableau VI) mais très faibles et pas significativement différentes de 0 au seuil de $5 \%$. En revanche, les corrélations de rang de Spearman entre moyennes de provenances (données à titre indicatif compte tenu du faible nombre de provenances) sont beaucoup 


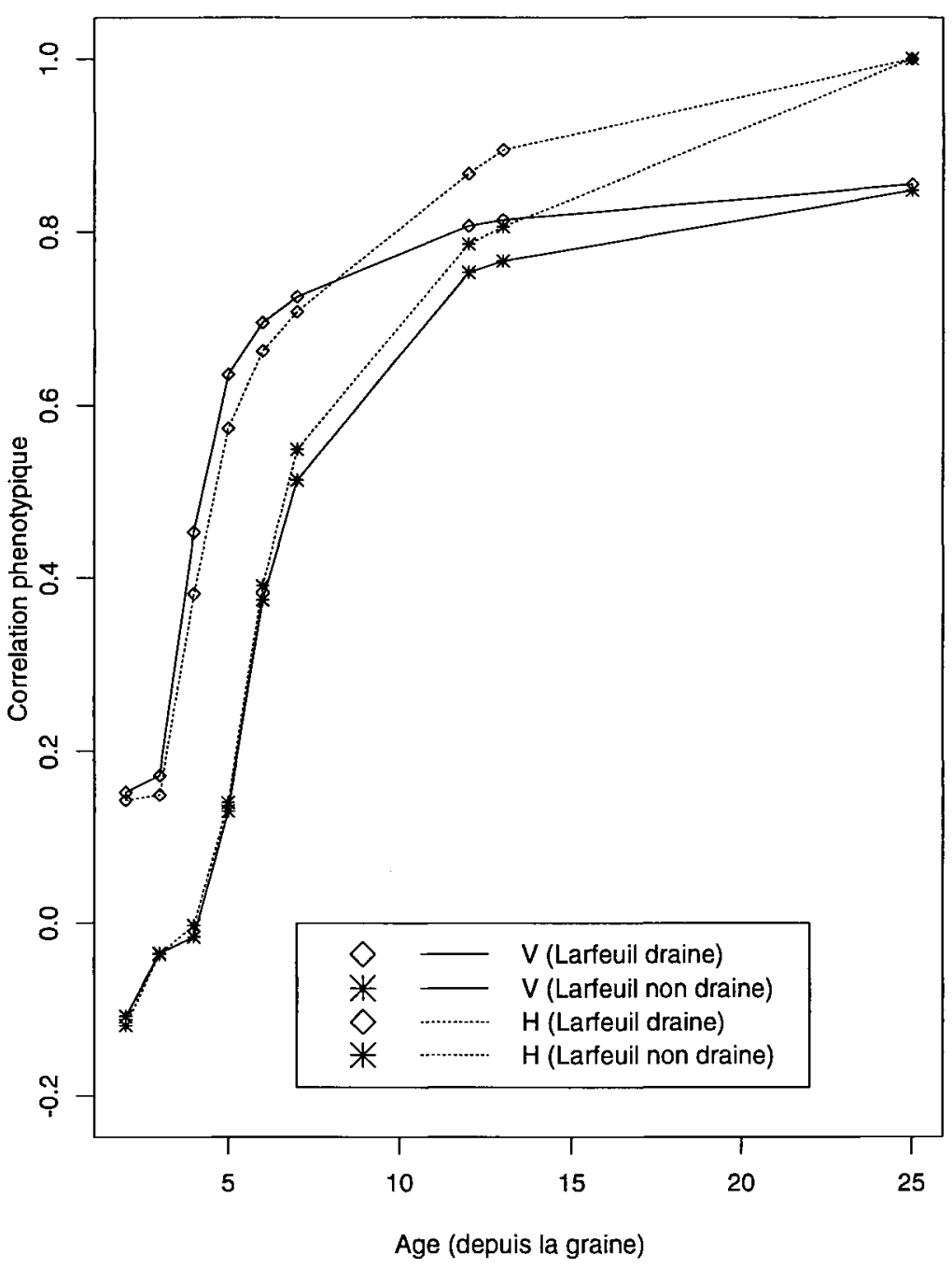

Fig 4. Évolution des coefficients de corrélation phénotypique entre la hauteur totale à un âge iet la hauteur totale $(\mathrm{H})$ et le volume individuel moyen $(\mathrm{V})$ de la tige à 25 ans.

plus fortes. Les liaisons sont défavorables à Larfeuil mais favorables à Épinal. À titre d'exemple, pour hauteur totale/rectitude, le coefficient de corrélation atteint $-0,29$ à Larfeuil : les provenances les plus vigoureuses sont mal classées pour la rectitude.

La liaison pénétration au pilodyn (Pi)infradensité (ID) est négative et significati- vement différente de 0 mais les niveaux de corrélation au niveau individuel à Larfeuil comme à Épinal sont assez faibles ( $r<$ $-0,35)$. En revanche, au niveau moyennes de provenances, la liaison n'est pas significativement différente de 0 : les coefficients de corrélation de rang égalent $+0,14^{\text {ns }}$ à Larfeuil et $-0,26^{\text {ns }}$ à Épinal. La mesure au 
pilodyn telle qu'elle a été prise sur écorce n'est donc qu'un assez mauvais indicateur de la valeur en infradensité. Une mesure de pénétration au pilodyn sous écorce eût été préférable.

Les corrélations entre hauteur totale $(H)$ et circonférence (C) d'une part et infradensité (ID) d'autre part sont négatives et significativement différentes de 0 , suggérant l'existence d'une liaison défavorable entre caractères de vigueur et de qualité du bois au niveau individuel. Les individus les plus vigoureux produisent un bois moins dense, à pénétration au pilodyn plus forte. En revanche, au niveau moyennes de provenance, la liaison reste positive et donc favorable entre caractères de vigueur et infradensité bien que non significativement différente de 0 . Par ailleurs, les arbres ayant la plus forte croissance en circonférence présentent également une plus forte proportion de duramen $(r>+0,4)$. Au niveau provenances, cette liaison n'est pas significativement différente de 0 .

\section{DISCUSSION ET CONCLUSION}

La décision a priori de limiter la zone d'origine des provenances à tester au sud-est de l'aire pouvait paraître très subjective compte tenu de l'immensité de l'aire naturelle du mélèze laricin et de sa variabilité. Néammoins, des résultats obtenus dans plusieurs dispositifs nord-américains (Michigan et Wisconsin) montrent une liaison très défavorable entre performances pour la vigueur d'une part et d'autre part, latitude et longitude d'origine (Riemenschneider et Jeffers, 1980 ; O'Connor et al, 1985). Les provenances les plus vigoureuses sont manifestement originaires du sud et de l'est de l'aire.

Des différences statistiquement significatives ont été mises en évidence dans cette étude entre provenances pour les principaux caractères étudiés. Les résultats sont cependant à considérer en fonction des sites et des caractères étudiés. Le site le plus discriminant pour l'ensemble des caractères est clairement le site drainé de Larfeuil. Le site non drainé apparaît peu propice à l'expression des différences de vigueur entre provenances et induit une forte hétérogénéité des performances intra-provenances ; le site d'Épinal est peu discriminant pour les caractères de qualité du bois.

D'autre part, il est intéressant de remarquer les faibles changements de rangs des provenances entre sites, et donc leur relative stabilité inter-sites pour les caractères principaux à l'exception des caractères de qualité du bois. Cette propriété avantageuse facilite ainsi le choix de provenances uniques pour le reboisement de sites aussi contrastés que ceux d'Épinal et de Larfeuil.

Bien que les liaisons soient souvent faibles et variables de site à site, il apparaît cependant difficile de trouver des provenances à forte croissance et bonne forme du fût. L'exemple de Garden of Eden Barren (GA-BA) est particulièrement illustratif : croissance excellente mais forme médiocre. En revanche, il semble qu'au niveau provenances forte vigueur et infradensité ne soient pas liées défavorablement. Koizumi et al (1992) montrent dans le même sens, à partir de provenances de mélèze du Japon, l'absence de liaisons défavorables entre caractères de vigueur et module d'élasticité de Young, paramètre bien corrélé à la densité du bois.

Mais comme l'observe Winkler (1973), la principale source de variabilité reste également dans cette étude le milieu de test (forts effets sites mais aussi forts effets blocs). La production serait selon cet auteur peu modifiée par les facteurs climatiques et l'altitude. Les plus fortes croissances sont obtenues sur des sols moyennement riches en éléments nutritifs, à nappe peu profonde et où les variations de niveau d'eau sont faibles. Le mélèze laricin peut valablement tirer parti de ces sols difficiles à mettre en valeur et sa production peut largement 
dépasser la production moyenne du mélèze d'Europe. À titre de rappel, les sites représentés dans cette étude peuvent être considérés comme marginaux pour l'épicéa commun lui-même et a fortiori pour les autres mélèzes. Dans la meilleure station à Épinal, le laricin atteint $13,5 \mathrm{~m}$ de hauteur totale à 25 ans, soit un niveau de productivité estimé à 50 ans de l'ordre de 9 à 10 $\mathrm{m}^{3} / \mathrm{ha} / \mathrm{an}$. Dans le site non drainé de Larfeuil à forte concurrence de molinie, le laricin a une croissance très médiocre avec une hauteur inférieure à $7 \mathrm{~m}$ et un niveau de productivité estimé inférieur à $4 \mathrm{~m}^{3} / \mathrm{ha} / \mathrm{an}$. Pour rappel, sur le même site, le pin à crochet utilisé en témoin atteint $5 \mathrm{~m}$ de hauteur totale.

Néanmoins, malgré sa bonne adaptation (survie élevée) et sa croissance satisfaisante dans ces stations marginales, le mélèze laricin souffre, quel que soit le site, de défauts de forme souvent sévères : nombreuses tiges flexueuses et fourchues. D'autre part, les provenances ayant la meilleure forme (Zone $5 \mathrm{E}$, Gogebic et Oneida) sont celles à croissance la plus faible. Il est par ailleurs intéressant de noter que ces 3 provenances sont plutôt originaires de la partie continentale de l'aire.

De ces résultats, il semble donc que l'intérêt majeur du mélèze laricin soit la possibilité de l'utiliser comme essence transitoire dans des stations à forte hydromorphie et à gelées tardives et de profiter de l'assèchement du sol qu'il provoque et de son couvert pour introduire des essences à plus grand intérêt économique mais moins rustiques. Dans ce cas, une préférence sera donnée aux provenances à croissance juvénile rapide plutôt qu'à celles avec une forme de fût satisfaisante. Une sélection précoce dans ce sens entre 7 et 12 ans des meilleures provenances a été montrée fiable. Garden of Eden Barren (NouvelleEcosse), Penobscot (Maine) ou encore Oneida (Wisconsin) pourraient dès lors être recommandées aux reboiseurs.
Bien que ce dispositif établi dès 1966 doive être considéré comme préliminaire, le faible nombre de provenances testées est évidemment contestable et ce d'autant que, comme le montrent O'Connor et al (1985), les différences de peuplement à peuplement peuvent être plus fortes encore que les différences entre régions de provenances. Un échantillonnage plus intensif des zones de provenances et peuplements et l'installation de nouveaux dispositifs pourraient être suggérés. Une autre approche sans doute plus attrayante serait la combinaison en hybridation inter-spécifique de la bonne tolérance aux sols hydromorphes du mélèze laricin et de la forte vigueur et bonne forme du fût de certains hybrides entre mélèzes d'Europe et du Japon (Pâques, 1992), suivie de la production en masse par bouturage du matériel sélectionné. Certains hybrides de ce type sont actuellement testés par l'INRA en France.

\section{REMERCIEMENTS}

Sont vivement remerciés pour leur aide lors des mesures sur le terrain MM $P$ Legroux et $M$ Vernier, chefs de domaine INRA, et leur équipe technique, ainsi que Mme $\mathrm{C}$ Schneider pour la gestion informatique des fichiers de données.

\section{RÉFÉRENCES}

Bachacou J, Masson JP, Miller C (1981) Manuel de la programmathèque statistique Amance 81. INRA, département de biométrie, $516 \mathrm{p}$

Bastien JC, Keller R (1980) Intérêts comparés du mélèze hybride (Larix $x$ eurolepis Henry) avec les deux espèces parentes. Rev For Fr 32, 521-526

Cech FC, Keys RN, Weingartner DH (1977) Seventhyear results of a tamarack provenance study. $I n$ : Proc 24th Northeastern Forest Tree Improvement Conference, Univ Maryland, College Park Maryland, July 26-29, 1976, 55-65

Cheliak WM, Wang J, Pitel JA (1988) Population structure and genic diversity in tamarack, Larix laricina (Du Roi) K Koch. Can J For Res 18, 1318-1324 
Erteld W (1962) Ertragstafelauszüge für den Gebrauch in der Praxis. Neumann Verlag, Leipzig, 105-115

Ferrand JC (1986) Mélèzes. Rev For Fr $n^{\circ}$ spécial 38 , 142-145

Fowells HA (1965) Tamarack. In : Silvics of forest trees of the United States. Agriculture Handbook $n^{\circ} 271$. US Depart of Agriculture, Forest Service, 227-234

Hamilton GJ, Christie JM (1971) Forest management tables (metric). Forestry Commission Booklet $n^{\circ} 34$, London, 142-145

Jeffers RM (1975) Survival and height growth of tamarack planted in northern Wisconsin. USDA Research note NC-190, North Central Forest Experiment Station, Saint Paul, MN, 1-3

Keylwerth R (1954) Ein Beitrag zür qualitativen Zuwachsanalyse, Holtz Roh Werkst 13, 77-83

Koizumi A, Takada K, Ueda (1992) Variation in modulus of elasticity among Japanese larch from different provenances. In : Proc IUFRO Centennial Meeting IUFRO Working Party S2.02-07 Berlin, Sept 5-12, 1992, 66-72

Martinsson O (1992) 30 years of provenance research on larch in Sweden. In: Proc IUFRO Centennial Meeting, IUFRO Working Party S2.02-07, Berlin, Sept 5-12, $1992,22-36$
Nassar R, Huhn M (1987) Studies on estimation of phenotypic stability: tests of significance for nonparametric measures of phenotypic stability. Biometrics $43,45-53$

O'Connor JM, Howe GT, Hanover JW (1985) Provenance variability in biomass production of Larix laricina in Michigan. In : Proc 4th North Central Tree Improvement Conference, East Lansing, MI, August $1985,92-101$

Pâques LE (1992) Performance of vegetatively propagated Larix decidua, $L$ kaempferi and $L$ laricina hybrids. Ann Sci For 49, 63-74

Périnot $\mathrm{CH}$ (1992) Intérêt du mélèze laricin pour le reboisement des zones tourbeuses. Rapport de stage BTS, École des Barres, Nogent-sur-Vernisson, $31 \mathrm{p}$

Riemenschneider DE, Jeffers RM (1980) Height and diameter of tamarack seed sources in northern Wisconsin. USDA Forest Service Research Paper NC190. North Central Forest Experiment Station, Saint Paul, MN, $6 \mathrm{p}$

Sokal RR, Rohlf FJ (1981) Biometry - The Principles and Practice of Statistics in Biological Research. Freeman, San Francisco, 2nd ed, $859 \mathrm{p}$

Winkler U (1973) Ergebnisse von Anbauversuchen der Niedersächsischen Forstlichen Versuchsanstalt mit Larix laricina (Koch). Allg Forst-u J Ztg 144, 25-31 